\title{
A PLASMON DRIVEN BOWSHOCK MODEL FOR THE NARROW LINE REGION OF NGC 5929
}

\author{
D. Taylor, J. E. Dyson \\ Department of Astronomy, \\ University of Manchester, \\ Manchester M13 9PL, U.K. \\ D. J. Axon, A. Pedlar \\ Nuffield Radio Astronomy Observatories, \\ Jodrell Bank, \\ Macclesfield, \\ Cheshire SK11 9DL, U.K.
}

Close links appear to exist between the optical narrow line region (NLR) in Seyfert galaxies and the nuclear non-thermal radio emission. Both occur on similar scales $\left(\sim 10^{2}-10^{3} \mathrm{pc}\right)$, and correlations have been found between the $21 \mathrm{~cm}$ radio power and both the [OIII] $\lambda 5007 \AA$ luminosity and linewidth (de Bruyn \& Wilson 1978; Whittle 1985). Approximate balance appears to exist between the thermal pressure of the NLR gas and the relativistic and magnetic pressure of the radio components (de Bruyn \& Wilson 1978; Unger et al. 1985; Pedlar, Dyson \& Unger 1985). There are also several examples of a direct spatial association between the optical and radio components, for example Mkn 78 (Pedlar et al. 1988).

A particularly good example for modelling this association is NGC 5929, a Type 2 Seyfert with a redshift of $2625 \mathrm{~km} \mathrm{~s}^{-1}$ (Whittle et al. 1986), corresponding to a distance of $53 \mathrm{Mpc}$ for $\mathrm{H}_{0}=50 \mathrm{~km} \mathrm{~s}^{-1} \mathrm{Mpc}^{-1}$. This galaxy has a simple two component structure in its nuclear region at both optical and radio wavelengths. Narrow band [OIII] $\lambda 5007 \AA$ imaging (Whittle et al. 1986) shows two core components with a separation of $\sim$ $1.7 \operatorname{arcsec}(\sim 190 \mathrm{pc})$ along PA $63 \pm 2^{\circ}$, on either side of the stellar nucleus. They are of unequal brightness, the stronger being to the south-west. Long-slit spectroscopic observations (Whittle et al. 1986) reveal two distinct [OIII] $\lambda 5007$ velocity components which, with the slit aligned along $\mathrm{PA} 60^{\circ}$, are also separated spatially. The components have heliocentric peak velocities of $2310 \pm 10 \mathrm{~km} \mathrm{~s}^{-1}$ (NE) and $2625 \pm 25 \mathrm{~km} \mathrm{~s}^{-1}$ (SW). By simply partitioning the line profiles into two portions about the median velocity of the two components, Whittle et al. (1986) derived their spatial separation to be $\sim 0.95 \operatorname{arcsec}(\sim 240 \mathrm{pc})$.

A $5 \mathrm{GHz}$ VLA map of the nuclear region (Ulvestad \& Wilson 1984) shows a two lobe radio structure; the components are unresolved and have a separation of $\sim 1.3$ arcsec in PA $\sim 60^{\circ}$. A VLBI observation (Whittle et al. 1986) gives an estimate of $10 \mathrm{pc}$ for the scale size of the lobes. 
We have developed a model for the NLR in which the linear motions of radioemitting bubbles of plasma ('plasmons') ejected from the Seyfert nucleus drive bowshocks into the ambient nuclear medium. The cooled shocked gas, accelerated by the plasmons and photoionised by the UV nuclear continuum, produces the optical NLR emission. This provides a natural explanation for the relationship between the radio continuum emission and the NLR, and avoids the severe problems of confinement inherent in the standard picture of the NLR as an assembly of clouds.

Our model consists of a steady state axisymmetric bowshock driven ahead of a plasmon which is moving at constant velocity through a homogeneous medium. The ambient gas is completely photoionised by continuum radiation from the Seyfert nucleus. This gas is compressed and heated on entering the shock, and then flows along the bowshock. As it does so it gradually cools into thermal equilibrium with the nuclear radiation field and finally merges back into the ambient medium. The deceleration of the plasmon by ram pressure is ignored and the flow pattern and plasmon shape hence assumed time independent. At any point on the bowshock surface each gas parcel is assumed to have a pressure equal to the ram pressure at that point and a velocity determined by global conservation of mass and momentum. Individual parcels of gas are assumed to cool independently, both radiatively and adiabatically. In this model, therefore, gas at a given distance from the apex of the bowshock comprises many individual packets each with a unique temperature, density and ionisation structure, but with the same pressure and velocity. The [OIII] emission from each point on the surface is calculated by summing the contributions from these elements. The radiative cooling law used in the calculation of the temperatures of the gas parcels is a numerical approximation to the non-equilibrium cooling curve of Falle (1975). To calculate the ionisation states of the gas elements, ionisation equilibrium is assumed at all times. This is justified for the purposes of calculating the optical emission as this is predominantly produced by cooled, photoionised gas.

The ionising UV radiation field of the galactic nucleus is modelled as a simple power law with spectral index $\alpha=1.4$, (defined so that the energy flux $f_{\nu} \propto \nu^{1-\alpha}$ ), which is typical of the Seyfert 2 galaxies observed by Ferland \& Osterbrock (1986).

The radio components in NGC 5929 are not resolved, so their shapes are unknown. However, one case of a Seyfert 2 galaxy where radio components are resolved is NGC 1068, which has a similar two component structure to NGC 5929, although on a larger scale. For want of a better guide to the bowshock shape to use in modelling NGC 5929, a Chebyshev fit was made to the outline of the NE radio lobe of NGC 1068 from the VLA map of Wilson \& Ulvestad (1983), and scaled down to the appropriate size.

We have modelled the NLR of NGC 5929 as a pair of radio plasmons of radius $\sim 10 \mathrm{pc}$ travelling in opposite directions away from the galactic nucleus, each producing a bowshock. We invoke an outflow in the ambient nuclear gas to produce the velocity separation between the two optical components, although this could equally be the result of galactic rotation.

A representative (but not unique) solution is sketched in Figure 1. The plasmon velocities are $550 \mathrm{~km} \mathrm{~s}^{-1}$ and $1020 \mathrm{~km} \mathrm{~s}^{-1}$ and the ambient medium has a density of $5 \mathrm{~cm}^{-3}$ and radial velocity $120 \mathrm{~km} \mathrm{~s}^{-1}$, resulting in shock velocities (at the stagnation points) of $430 \mathrm{~km} \mathrm{~s}^{-1}$ and $900 \mathrm{~km} \mathrm{~s}^{-1}$ respectively. 


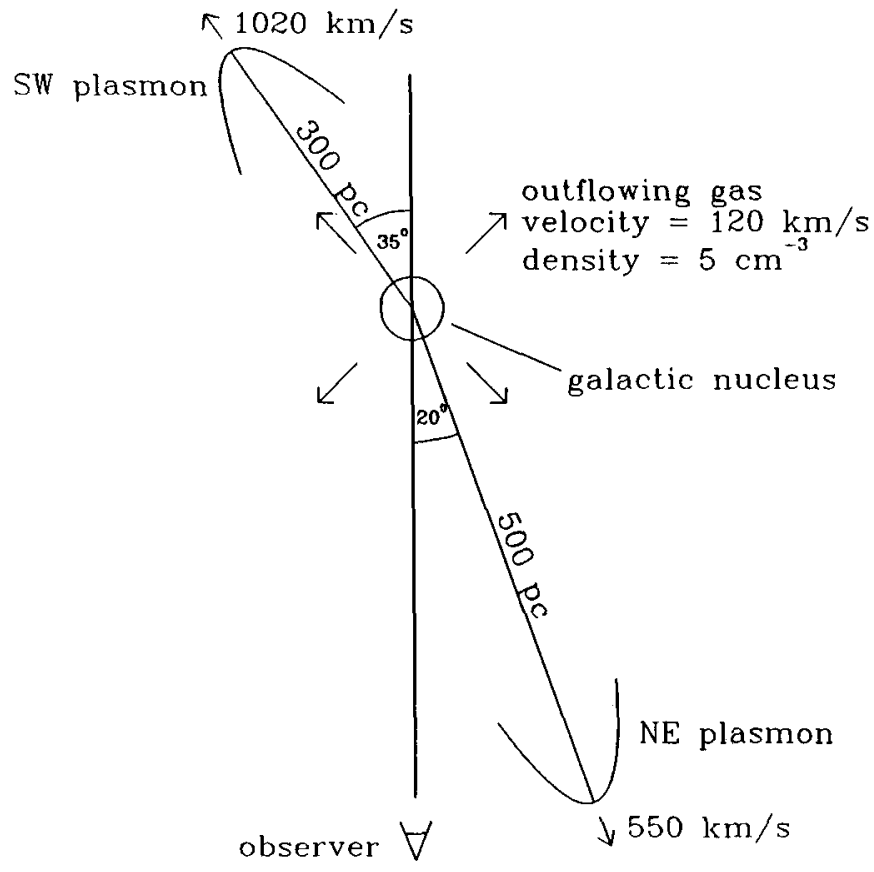

FIGURE 1: Sketch of the plasmon model for NGC 5929.

Figure 2 shows the sequence of [OIII] $\lambda 5007$ line profiles from Whittle et al. (1986) with the slit aligned along PA $60^{\circ}$, and the equivalent profiles produced by the model. To obtain these, the bowshock emission was convolved with a gaussian of FWHM $400 \mathrm{pc}$ to simulate the effects of seeing, and the profiles convolved with a $45 \mathrm{~km} \mathrm{~s}^{-1} \mathrm{FWHM}$ gaussian to account for instrumental broadening. Figure 3 shows the [OIII] component intensity distribution from Whittle et al. (1986) along with that produced here. Table 1 lists the observable parameters of the model, and the corresponding values from Whittle et al. (1986).

TABLE 1: [OIII] component properties produced by the plasmon model and the corresponding observational values (from Whittle et al. 1986).

\begin{tabular}{|c|c|c|c|}
\hline & $0.95 \pm 0.11$ & 1.19 \\
\hline & Velocity difference $(\mathrm{km} / \mathrm{s})$ & $315 \pm 18$ & 325 \\
\hline \multirow[t]{2}{*}{ FWHM $(\mathrm{km} / \mathrm{s})$} & NE & $80 \pm 10$ & 96 \\
\hline & SW & $185 \pm 20$ & 184 \\
\hline \multirow[t]{2}{*}{$L_{5007}(\mathrm{erg} / \mathrm{s})$} & NE & $1.4 \times 10^{40}$ & $9.8 \times 10^{39}$ \\
\hline & sW & $3.0 \times 10^{40}$ & $3.2 \times 10^{40}$ \\
\hline
\end{tabular}



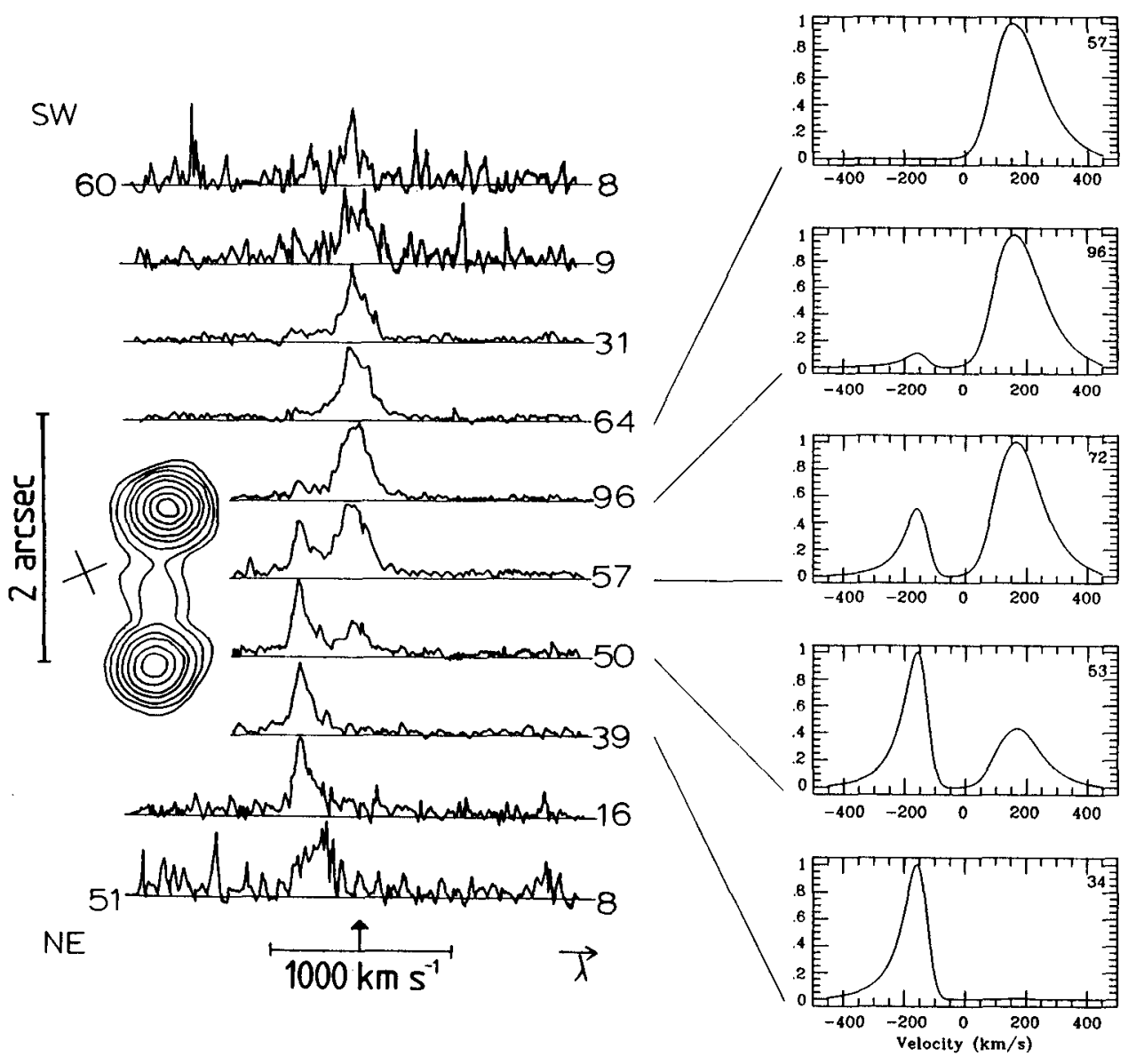

FIGURE 2: Observational and theoretical [OIII] $\lambda 5007$ line profiles for NGC 5929. The observations are from the long-slit data of Whittle et al. (1986). Spatial increments along the slit are 0.63 arcsec long and the increment numbers run from 51 (NE) to 60 (SW). The profiles are autoscaled, a relative intensity scale is given by the numbers to the right of each spectrum, which are proportional to the peak intensity of that spectrum. The vertical arrow at the bottom indicates a reference velocity of $2625 \mathrm{~km} \mathrm{~s}^{-1}$ (the galactocentric redshift of NGC 5929). Superimposed at the same scale and orientation is a radio map, taken from Ulvestad and Wilson (1984). The cross marks the position of the stellar nucleus. The profiles generated by the plasmon model correspond to slit increments 53 to 57 . The velocities are relative to the galactic nucleus. The profiles are autoscaled, with the relative intensities of each spectrum given by the number in the top right hand corner. 


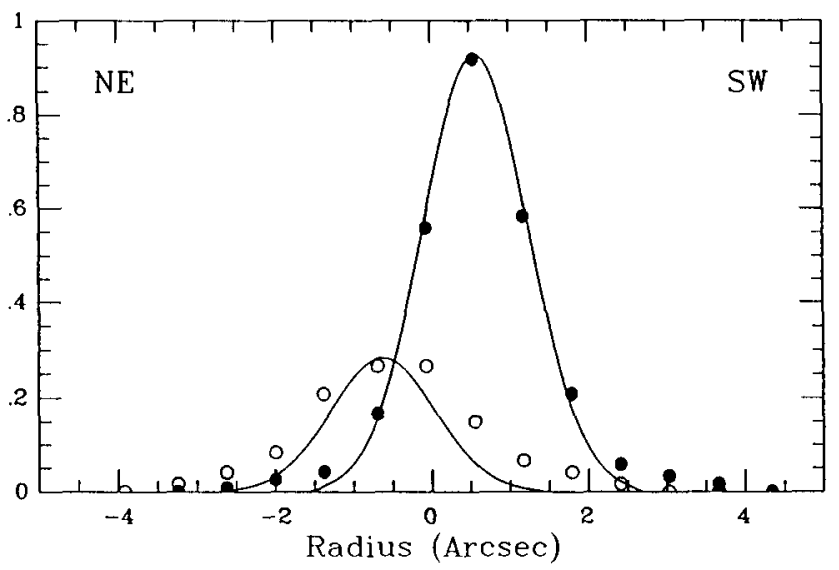

FIGURE 3: [OIII] component intensity distribution of the plasmon model. The points represent the Gaussian fits to long-slit data made by Whittle et al. (1986), with the open circles corresponding to the NE component and the filled circles to the SW component. The solid lines show the distributions produced by the model. The horizontal axis represents the distance from the position of the galactic nucleus in the model.

\section{REFERENCES}

De Bruyn, A. J. \& Wilson, A. S., 1978. Astron. Astrophys., 64, 433.

Falle, S. A. E. G., 1975. Astron. Astrophys., 42, 323.

Ferland, G. J. \& Osterbrock, D. E., 1986. Astrophys. J., 300, 658.

Pedlar, A., Dyson, J. E. \& Unger, S. W., 1985. Mon. Not. R. astr. Soc., 214, 463.

Pedlar, A., Meaburn, J., Axon, D. J., Unger, S. W., Guerrine, N., Whittle, M., Meurs, E. \& Ward, M., 1989. Mon. Not. R. astr. Soc., 238, 863.

Ulvestad, J. S. \& Wilson, A. S., 1984. Astrophys. J., 285, 439.

Unger, S. W., Pedlar. A., Booler, R. V. \& Harrison, B. A., 1985. Mon. Not. R. astr. Soc., 219, 387.

Whittle, M., 1985. Mon. Not. R. astr. Soc., 213, 33.

Whittle, M., Haniff, C. F., Ward, M. J., Meurs, E. J. A., Pedlar, A., Unger, S. W., Axon, D. J. \& Harrison, B. A., 1986. Mon. Not. R. astr. Soc., 222, 189.

Wilson, A. S. \& Ulvestad, J. S., 1983. Astrophys. J., 275, 8.

\section{Discussion:}

OSTERBROCK: You have presented this model for NGC 5929. Do you consider it as more generally valid to many or all Seyfert 2 galaxies?

TAYLOR: Yes. Optical NLR emission appears to be closely related to the radio emission in Seyfert galaxies, and could be a result of the interaction between radio components and the interstellar medium. Detailed modelling, however, is only possible in the very few cases where the optical structure can be resolved. 